\title{
Statistical Atlas-Based Detection of Abnormalities in Brain Perfusion: Comparing Models and Estimating Detection Performance
}

\author{
Torbjørn Vik ${ }^{1,2}$, Fabrice Heitz ${ }^{1}$, and Jean-Paul Armspach ${ }^{2}$ \\ 1 LSIIT UMR-7005 CNRS / Strasbourg I University, France \\ 2 IPB UMR-7004, CNRS / Strasbourg I University, France
}

\begin{abstract}
When developing statistical models of normal brain perfusion, two questions are of crucial interest: How well does an atlas describe normality and how sensitive is it at detecting abnormalities? In this work we attempt to find partial responses to these questions by extensively experimenting with our local database of SPECT images of normal subjects, systematically synthesizing abnormalities and using a leave-one-out scheme to estimate the combined detection and modeling performance. Having such an evaluation framework we are then able to compare different models. We have tested three voxel-based models and we present results showing that a principal component analysis (PCA) model with one or two principal components outperforms a model based uniquely on the voxel-mean and -variance for small abnormalities. A robust version of the PCA model, specially designed for abnormalities of large size, does not bring significant gain over the other methods.
\end{abstract}

\section{Introduction}

A probabilistic atlas of brain perfusion is a statistical representation of localization and intensities of perfusion patterns in a population. We are interested in creating a brain atlas based on SPECT images representing a general normal population and that could later be specialized for studies of epilepsy and dementia. We have chosen, in a first approach, to work on voxel-based approaches similar to statistical parametric mapping (SPM) 1. We consider three different models, a local model, based on the voxel-mean and-variance, a global model, based on principal component analysis (PCA), and a robust version of the latter, useful in the case of large zones of abnormality.

The performance of an atlas is in general limited by the noise in the imaging technique, model approximation as well as problems related to the representativity of images used in the atlas construction process (learning base images) e.g. are the supposed normal subjects really normal and do they have a normal brain perfusion pattern at the moment of scanning? Furthermore, and more specific to our voxel-based approaches, imperfect spatial normalization and other preprocessing steps will lead to learning artifacts. In our approach, all these factors lead to modeling errors and we need methods that are able to evaluate how 
accurate our model assumptions actually are and how much confidence we can put in our atlas when detecting abnormalities.

In this work we present an experimental assessment of three statistical atlasbased approaches for the detection of abnormalities of brain perfusion. The validation is conducted by systematically simulating zones of hyperperfusion and using a leave-one-out strategy to evaluate performance. This approach does not generate simulations that favor one model over another [2]. We define a quality metric for summarizing performance and present the results as receiver operating characteristics (ROCs). Other simulation studies for assessing cerebral lesions in SPECT images have been described in the literature. In [3], the authors decrease and increase the perfusion on a sphere in the right frontal lobe of the mean image to evaluate the capacity of SPM to detect changes. We think this approach yields an optimistic estimate because an abnormality can vary in an additive manner around the variation of normal images, not only around their mean. Furthermore, the study is limited to only one location of the abnormality whereas we show in this article that the detection quality depends on location. Another study [4 adds inclusions to a software phantom, thereby simulating a single-subject activation study, whereas we are more concerned with multiple individuals.

\section{Methods and Materials}

\subsection{Database of Normal Subjects}

Our database contains 21 ECD-SPECT (double-headed camera with parallel collimator, filtered back-projection with FWHM of 8mm) and MRI (1mm) images of normal, healthy volunteers at rest ( 7 males, 14 females, two groups aged $25.1 \pm 6.7$ and $46.9 \pm 4.3$ years were pooled together). All the MRI images were smoothed and the SPECT images as well as the MRI images were resampled to $4 \mathrm{~mm}$ resolution. The SPECT images were all registered to their MRI counterparts using a rigid transformation model and Woods similarity criterion [5]. For spatial normalization, all the MRI images were registered to the brain template 1 proposed by the international consortium of brain mapping (ICBM452) using a 12 parameter affine transformation. Composing these transformations to obtain the final transformation, the original SPECT images were brought into the template reference system using trilinear interpolation. To segment the brain from the head in the SPECT images, we simply used Otsu-thresholding and an algorithm to fill the remaining holes. Finally, we normalized the brains to have the same total number of brain counts. The whole chain of preprocessing is automatic.

\subsection{Synthesized Images}

The abnormalities to be detected in SPECT images vary in shape, size, intensity, intensity-profile and position (location in the brain). We consider only hyperperfusions in a first time and model these as inclusions that we add to an image

\footnotetext{
${ }^{1}$ http://www.loni.ucla.edu/ICBM/ICBM_ICBMAtlases.html
} 
from the test set. This yields what we call a test image and is evaluated by the detection algorithm to yield the tested image. To evaluate performance, we consider this inclusion to be the ground truth: it should be the only thing a detection algorithm detects. Many false alarms thus means that the model has reduced capacity of describing normality and the measured performance is therefore a combined measure of detection capacity and the capability of describing normality. Note that since there may be zones in a normal image that are effectively hyperperfused, but not abnormal, these may be detected and evaluated as false alarms even though they are actually not. This means we may be underestimating the performance of a model, yielding a worst case performance. The method is fair at comparing different models though.

We have performed two different experiments. In the first, we have used inclusions of moderate size to compare the local model with the global model (see 2.3). Here we used inclusions with intensity of $15 \%$ and $25 \%$ of the average brain intensity. Each inclusion consisted of a constant intensity sphere with a diameter of $20 \mathrm{~mm}$ and was filtered with a Gaussian kernel. This filtered inclusion was thresholded at $2 / 3$ of the intensity value to define the support of the ground truth used for evaluation. The ground truth for these inclusions counted 57 voxels, whereas the whole brain mask used counted 17877 voxels. The inclusions were successively positioned at 16 different locations to cover the brain evenly, 6 in each hemisphere and 4 in the hemisphere separating plane. An example of a test image is given in Fig. 1 In a second experiment we wanted to evaluate the effect of larger inclusions. We used inclusions with diameter sizes of $44 \mathrm{~mm}$ (751 voxels) and $68 \mathrm{~mm}$ (2601 voxels) before filtering, as well as intensities of $25 \%$ and $35 \%$ of the average brain level. The same locations as in the first experiment were used. Since these larger inclusions sometimes covered space outside the brain, their actual size were mostly lower than the values indicated above. In future work, we plan to extend the experiments to use more specific inclusions.

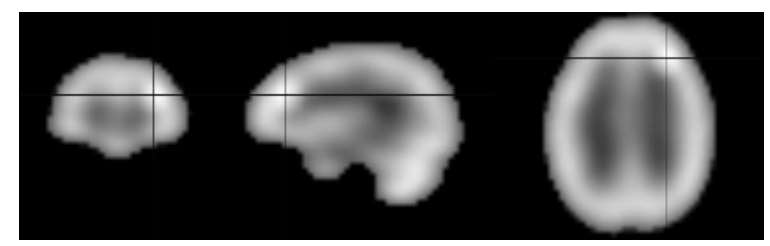

Fig. 1. A coronal, sagittal and axial view of a test image with a cursor marking the center of the inclusion (diameter: $20 \mathrm{~mm}$, intensity: $25 \%$ of the average brain level)

\subsection{Models}

For modeling purpose we associate each image with a vector by lexicographically ordering the voxels inside a common brain mask into a vector. This mask is taken as the intersection of all (segmented) brains in the learning set. We consider 
three models that can all be derived from the following linear image generative model [6]

$$
\boldsymbol{y}=\boldsymbol{W} \boldsymbol{x}+\boldsymbol{\mu}+\boldsymbol{\epsilon},
$$

relating a low dimensional latent variable $\boldsymbol{x}$ with the high dimensional image $\boldsymbol{y}$ by means of the generation matrix $\boldsymbol{W}$. The vector $\boldsymbol{\mu}$ is the model mean and $\boldsymbol{\epsilon}$ is the voxel noise, the mean image $\boldsymbol{\mu}$ is estimated as the learning set average. In the first model, which we refer to as the local model, the variations around the mean image is not taken into account $(\boldsymbol{W}=\mathbf{0})$ and the noise is considered to be Gaussian, $\boldsymbol{\epsilon} \sim \mathcal{N}(0, \boldsymbol{\Psi})$ with $\boldsymbol{\Psi}$ diagonal. This means that the model is entirely defined by the voxel-mean and -variance. The variance $\boldsymbol{\Psi}$ is estimated from the $N$ learning set residuals $\boldsymbol{\epsilon}^{(j)}=\boldsymbol{y}^{(j)}-\boldsymbol{\mu}, j=1, \ldots, N$ as $(\boldsymbol{\Psi})_{i}=\left(\frac{1}{N-1} \sum_{j} \boldsymbol{\epsilon}^{(j)} \boldsymbol{\epsilon}^{(j) T}\right)_{i}$, where the subscript $i$ is the $i$ th diagonal element (voxel). Abnormalities are detected by comparing at each voxel the z-score $\boldsymbol{t}=\boldsymbol{\Psi}^{-1 / 2}(\boldsymbol{y}-\boldsymbol{\mu})$ against a threshold. The z-score $\boldsymbol{t}$ can be related to the probability of observing the residual $\boldsymbol{\epsilon}=\boldsymbol{y}-\boldsymbol{\mu}$.

The second model is the same PCA model as in [7], where the $q$ column vectors of $\boldsymbol{W}$ are the $q$ first (normalized) principal components of the (meanfree) learning set images and $p(\boldsymbol{\epsilon})$ is again considered to be Gaussian. From an observed image $\boldsymbol{y}$, the corresponding latent variable $\boldsymbol{x}$ is found by maximum likelihood (ML) estimation, which is equivalent to the least squares (LS) estimate of $\boldsymbol{x}$ and yields $\hat{\boldsymbol{x}}_{G M L}=\boldsymbol{W}^{T}(\boldsymbol{y}-\boldsymbol{\mu})$. The tested image is defined as the z-score of the residual and is calculated as $\boldsymbol{t}=\boldsymbol{\Psi}^{-1 / 2}\left(\boldsymbol{y}-\boldsymbol{\mu}-\boldsymbol{W} \hat{\boldsymbol{x}}_{G M L}\right)$. The noise variance $\boldsymbol{\Psi}$ is estimated from the learning residuals $\boldsymbol{\epsilon}^{(j)}=\boldsymbol{y}^{(j)}-\boldsymbol{\mu}-\boldsymbol{W} \hat{\boldsymbol{x}}_{G M L}^{(j)}$ as above: $(\boldsymbol{\Psi})_{i}=\left(\frac{1}{N-1-q} \sum_{j} \boldsymbol{\epsilon}^{(j)} \boldsymbol{\epsilon}^{(j) T}\right)_{i}$.

The third model that we have considered is a robust version of the above PCA model where we model $p(\boldsymbol{\epsilon})$ as non-Gaussian. This is a more realistic model for large inclusions of high intensity since these represent outliers to the Gaussian distribution. LS estimates are known to be sensitive to such outliers and one approach to reduce this sensitivity is to replace the quadratic function in the exponential of the Gaussian by a robust cost function $\rho(\cdot)$, that down-weights the contribution of the outliers [8]. With this model the ML estimate of $\boldsymbol{x}$ is obtained as

$$
\hat{\boldsymbol{x}}_{R M L}=\arg \max _{\boldsymbol{x}} \exp \left(-\frac{1}{2} \sum_{i} \rho\left(\epsilon_{i}\right)\right)
$$

and must be estimated iteratively. The tested image is again defined as $\boldsymbol{t}=$ $\boldsymbol{\Psi}^{-1 / 2}\left(\boldsymbol{y}-\boldsymbol{\mu}-\boldsymbol{W} \hat{\boldsymbol{x}}_{R M L}\right)$.

For the PCA models the number of principal components $q$ must be specified. Too few components limit description power and too many may result in overfitting [9], both cases leading to a model less powerful in describing normality. We have done our experiments with 1-4 principal components.

\subsection{Leave-One-Out}

The leave-one-out methodology is a standard method in pattern recognition to estimate recognition and modeling performance when one has only a dataset of small size [9]. Given a dataset of size $N$, leave-one-out simply consists of splitting 
the imageset in a set of $N-1$ training images and one test image. The training set is used to estimate model parameters and then the model is used to evaluate the test image. The procedure is subsequently repeated for all $N$ images and the results averaged.

\subsection{Calculation of ROCs}

To calculate the receiver operating characteristics (ROC) 29], we have to define the true detection rate (TDR) and the false detection rate (FDR) (or equivalently sensitivity and specificity). We calculate these as probabilities. The tested images are thresholded at different levels to obtain binary images from which we calculate the TDR and the FDR. By varying the threshold level we obtain the ROC curve. Let $\delta_{k}^{(j)}$ take on the value 1 for detection at voxel $k$ in subject $j=1, \ldots N$ and 0 for non-detection and let $I$ designate the $3 \mathrm{D}$ region defined by the inclusion. We define the TDR and FDR as:

$$
\mathrm{TDR}=\frac{\sum_{j=1}^{N} \sum_{k \in I} \delta_{k}^{(j)}}{\sum_{j=1}^{N} \sum_{k \in I} 1} \quad \text { and } \quad \mathrm{FDR}=\frac{\sum_{j=1}^{N} \sum_{k \in B \backslash I} \delta_{k}^{(j)}}{\sum_{j=1}^{N} \sum_{k \in B \backslash I} 1} \text {, }
$$

where $B$ is the region defined by the brain (we use the same brain mask as for PCA). We do not correct the results for multiple comparisons as in SPM.

\section{Results}

In the first experiment involving small inclusions, using Eq. (11) and the leaveone-out methodology, we obtained the average ROC curves in Fig. 2 We see that all the PCA models perform better than the local model, whereas the PCA models are quite similar. A more detailed analysis show that the PCA1 model is the better for FDR $<0.006$ and thereafter the PCA2 model is the best. It is interesting to notice that the PCA3 and PCA4 models are inferior to the PCA1 and PCA2 models, maybe indicating an overfitted model. Examples of tested images can be seen in Fig. 3 In this experiment the robust PCA model yielded almost exactly the same results as the PCA model and is therefore not depicted.

The curves in Fig. 2 have been calculated by averaging all locations. Since this may hide information about location-dependent sensitivity, we have also calculated the ROC curves by averaging each location across all individuals. These are shown in Fig. 4 and show significant differences depending on the location of inclusions.

From the second experiment, a result from using the large inclusions is shown in Fig. 5. The performance of the PCA2 model has dropped significantly as compared to the other models, whereas the robust PCA2 model, close to the PCA1 models, exhibit the best performance. This may be explained by the global nature of the PCA model: the LS estimation of the latent variable $\boldsymbol{x}$ has been corrupted by the large inclusion, thereby falsifying the reconstructed image $\hat{\boldsymbol{y}}$ and the corresponding tested images. The model with two principal component is more affected than the model with one component whereas the local model is as 


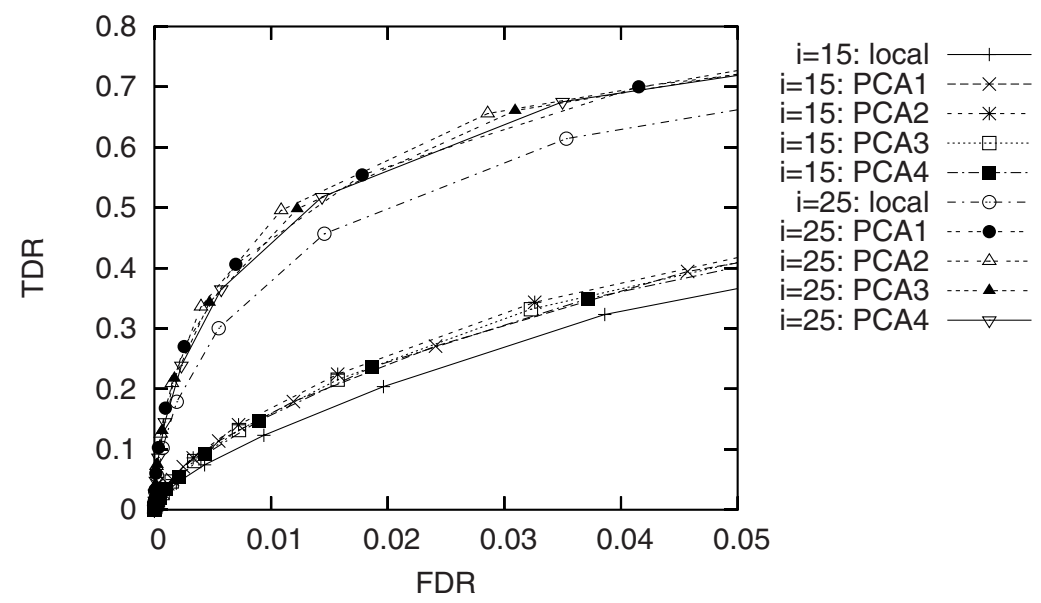

Fig. 2. ROC curves for FDR $\in[0,0.05]$ and TDR $\in[0,0.8]$, obtained as average over all locations and all tested images. The inclusions had diameter of $20 \mathrm{~mm}$ and intensities of $15 \%(l=15)$ and $25 \%(l=25)$ of the average brain level. The PCA models are denoted "PCA $n$ ", where $n$ is the number of principal components used. For these inclusions, robust PCA yield the same results as standard PCA

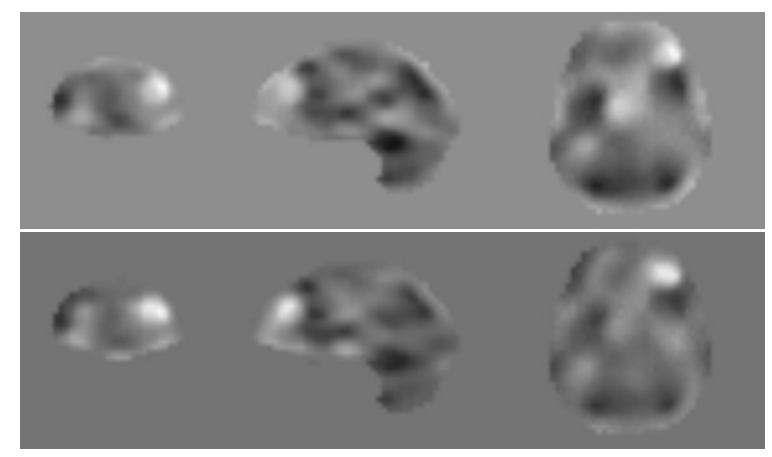

Fig. 3. Tested images of the image in Fig. 1 as calculated by the models. Up: Local model. Down: PCA2 model. These are example images from which we calculate the ROC curves. The ROC curves are statistical measures of performance and, as seen in Fig. 2, the global models perform better than the local model

expected unaffected. The results obtained with inclusions with size of maximum 751 voxels showed a small improvement of the robust with respect to non-robust PCA models, though all significantly above the local model. Here again the PCA models with one and two components performed better than PCA models with three and four components. 


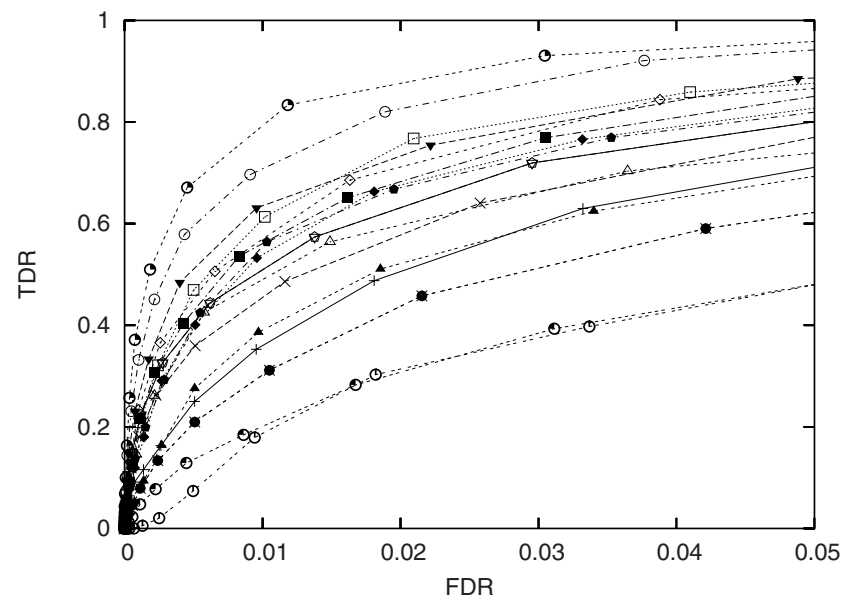

Fig. 4. ROC curves for FDR $\in[0,0.05]$ of each location examined in the study, averaged over all tested images. Results obtained with the PCA2 model, inclusion diameter of $20 \mathrm{~mm}$ and intensity of $25 \%$ of the average brain level

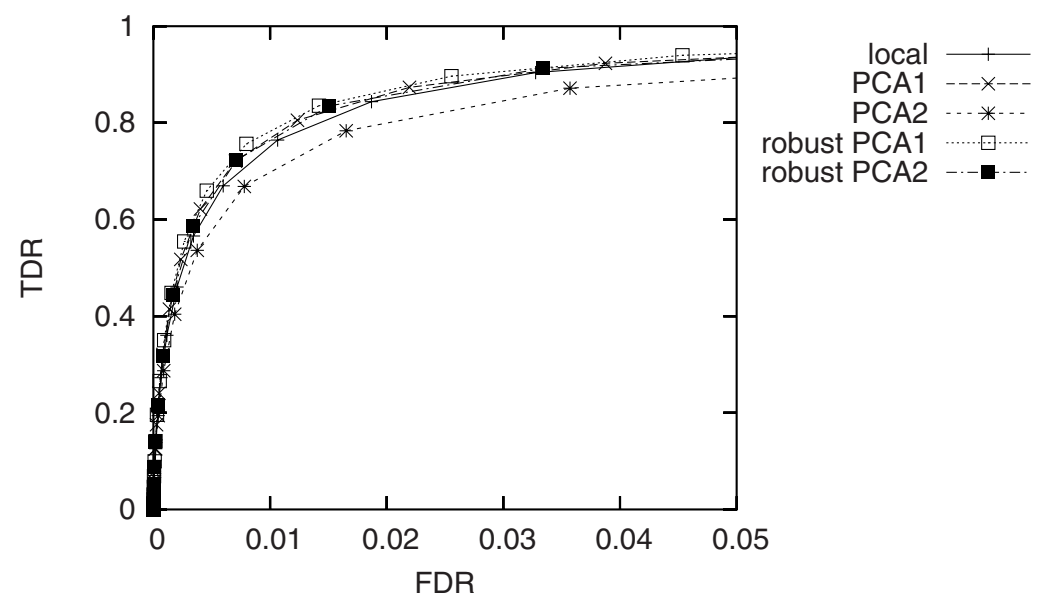

Fig. 5. ROC curves for FDR $\in[0,0.05]$, obtained as average over all locations with inclusion size of maximum 2601 voxels and intensity of $35 \%$ of average brain level

\section{Conclusion and Discussion}

Our experiments have shown that a PCA-based model outperforms a local model for the detection of small abnormalities of brain perfusion. However, for large abnormalities, due to its global nature, the PCA detection performance drops (even below that of the local model for very large inclusions). This can be par- 
tially remedied by replacing it by a robust version. The variation in detection performance depending on the location must be further analyzed and quantified, future work includes finding ways of exploiting this information. The validation measure we propose is limited in the sense that a physicist will evaluate the detected abnormal patterns at different thresholds using a priori, spatial and other supplementary knowledge in his diagnosis.

\section{References}

1. Friston, K.J., Holmes, A.P., Worsley, K.J., Poline, J.P., Frith, C.D., Frackowiak, R.S.J.: Statistical parametric maps in functional imaging: A general linear approach. Human Brain Mapping 2 (1995) 189-210

2. Bowyer, K.W.: Validation of medical image analysis techniques. In Sonka, M., Fitzpatrick, J.M., eds.: Handbook of Medical Imaging. SPIE Press (2000) 567-607

3. Stamatakis, E.A., Glabus, M.F., Wyper, D.J., Barnes, A., Wilson, J.T.L.: Validation of statistical parametric mapping (SPM) in assessing cerebral lesions: A simulation study. NeuroImage 10 (1999) 397-407

4. Lahorte, P., Vandenberghe, S., Laere, K.V., Audenaert, K., Lemahieu, I., Dierckx, R.A.: Assessing the performance of SPM analyses of SPECT neuroactivation studies. NeuroImage 12 (2000) 757-764

5. Woods, R., Cherry, S., Mazziotta, J.: Rapid automated algorithm for aligning and reslicing PET images. J Comput Assist Tomogr 16 (1992) 620-633

6. Tipping, M.E., Bishop, C.: Probabilistic principal component analysis. Journal of the Royal Statistical Society, Series B 61 (1999) 611-622

7. Houston, A.S., Kemp, P.M., Macleod, M.A.: A method for assessing the significance of abnormalities in HMPAO brain SPECT images. The Journal of Nuclear Medicine 35 (1994) 239-244

8. Black, M.J., Jepson, A.D.: EigenTracking: Robust matching and tracking of articulated objects using a view-based representation. International Journal of Computer Vision 26 (1998) 63-84

9. Duda, R.O., Hart, P.E., Stork, D.G.: Pattern Classification. 2 edn. John Wiley \& Sons (2001) 